\title{
An X\#Ray, Infrared, and Submillimeter Flare of Sagittarius A*
}

\section{Citation}

Marrone, D. P., F. K. Baganoff, M. R. Morris, J. M. Moran, A. M. Ghez, S. D. Hornstein, C. D. Dowell, et al. 2008. "An X\#Ray, Infrared, and Submillimeter Flare of Sagittarius A*." Astrophysical Journal 682 (1) (July 20): 373-383. doi:10.1086/588806.

\section{Published Version}

doi:10.1086/588806

\section{Permanent link}

http://nrs.harvard.edu/urn-3:HUL.InstRepos:11596977

\section{Terms of Use}

This article was downloaded from Harvard University's DASH repository, and is made available under the terms and conditions applicable to Other Posted Material, as set forth at http:// nrs.harvard.edu/urn-3:HUL.InstRepos:dash.current.terms-of-use\#LAA

\section{Share Your Story}

The Harvard community has made this article openly available.

Please share how this access benefits you. Submit a story.

\section{Accessibility}




\title{
AN X-RAY, INFRARED, AND SUBMILLIMETER FLARE OF SAGITTARIUS A*
}

\author{
D. P. Marrone, ${ }^{1,2}$ F. K. Baganoff ${ }^{3}$ M. R. Morris, ${ }^{4}$ J. M. Moran, ${ }^{5}$ A. M. Ghez, ${ }^{4,6}$ S. D. Hornstein, ${ }^{7}$ \\ C. D. Dowell,${ }^{8}$ D. J. Muñoz, ${ }^{5}$ M. W. Bautz,${ }^{3}$ G. R. Ricker, ${ }^{3}$ W. N. Brandt, ${ }^{9}$ G. P. Garmire, ${ }^{9}$ \\ J. R. Lu, ${ }^{4}$ K. Matthews, ${ }^{10}$ J.-H. Zhao, ${ }^{5}$ R. Rao, ${ }^{11}$ and G. C. Bower ${ }^{12}$ \\ Received 2007 December 17; accepted 2008 April 4
}

\begin{abstract}
Energetic flares are observed in the Galactic supermassive black hole Sagittarius A* from radio to X-ray wavelengths. On a few occasions, simultaneous flares have been detected in IR and X-ray observations, but clear counterparts at longer wavelengths have not been seen. We present a flare observed over several hours on 2006 July 17 with the Chandra X-Ray Observatory, the Keck II telescope, the Caltech Submillimeter Observatory, and the Submillimeter Array. All telescopes observed strong flare events, but the submillimeter peak is found to occur nearly 100 minutes after the X-ray peak. Submillimeter polarization data show linear polarization in the excess flare emission, increasing from $9 \%$ to $17 \%$ as the flare passes through its peak, consistent with a transition from optically thick to thin synchrotron emission. The temporal and spectral behavior of the flare require that the energetic electrons responsible for the emission cool faster than expected from their radiative output. This is consistent with adiabatic cooling in an expanding emission region, with X-rays produced through self-Compton scattering, although not consistent with the simplest model of such expansion. We also present a submillimeter flare that followed a bright IR flare on 2005 July 31. Compared to 2006, this event had a larger peak IR flux and similar submillimeter flux, but it lacked measurable X-ray emission. It also showed a shorter delay between the IR and submillimeter peaks. Based on these events we propose a synchrotron and self-Compton model to relate the submillimeter lag and the variable IR/X-ray luminosity ratio.
\end{abstract}

Subject headings: black hole physics — Galaxy: center — polarization

Online material: color figures

\section{INTRODUCTION}

The radio, IR, and X-ray source Sagittarius $A^{*}$ is associated with a supermassive black hole at the center of our Galaxy (Melia \& Falcke 2001). Spectral measurements at all wavelengths where Sgr $\mathrm{A}^{*}$ is not hidden by confusion or Galactic absorption show it to be extremely underluminous for its mass, radiating just $10^{-9} L_{\text {Edd }}$. A variety of physical models have been shown to adequately reproduce the quiescent spectrum of $\mathrm{Sgr}^{*}$ (e.g., Falcke \& Markoff 2000; Melia et al. 2001; Yuan et al. 2003). Discrimination between the proposed accretion and outflow models will require information complementary to the spectral data.

Since the discovery of X-ray and IR flares in Sgr A* (Baganoff et al. 2001; Genzel et al. 2003; Ghez et al. 2004), transient events

\footnotetext{
1 Jansky Postdoctoral Fellow, National Radio Astronomy Observatory.

2 Kavli Institute for Cosmological Physics, University of Chicago, 5640 South Ellis Avenue, Chicago, IL 60637; dmarrone@uchicago.edu.

3 Kavli Institute for Astrophysics and Space Research, Massachusetts Institute of Technology, Cambridge, MA 02139-4307.

4 Department of Physics and Astronomy, University of California, Los Angeles, CA 90095-1547.

5 Harvard-Smithsonian Center for Astrophysics, 60 Garden Street, Cambridge, MA 02138.

6 Institute for Geophysics and Planetary Physics, University of California, Los Angeles, CA 90095-1565.

7 Center for Astrophysics and Space Astronomy, Department of Astrophysical and Planetary Sciences, University of Colorado, Boulder, CO 80309.

8 Jet Propulsion Laboratory, California Institute of Technology, MS 169-506, 4800 Oak Grove Drive, Pasadena, CA 91109.

9 Department of Astronomy and Astrophysics, Pennsylvania State University, University Park, PA 16802-6305.

${ }_{10}$ Caltech Optical Observatories, California Institute of Technology, MS 320-47, Pasadena, CA 91125.

11 Institute of Astronomy and Astrophysics, Academia Sinica, P.O. Box 23-141, Taipei 10617, Taiwan.

12 Department of Astronomy and Radio Astronomy Laboratory, University of California at Berkeley, Campbell Hall, Berkeley, CA 94720.
}

have been studied extensively. Such observations have found that Sgr $\mathrm{A}^{*}$ is highly variable, with increases in X-ray luminosity of up to 160 times over the quiescent emission (Porquet et al. 2003) and smaller flares on hour timescales at longer wavelengths (e.g., Ghez et al. 2004; Mauerhan et al. 2005; Herrnstein et al. 2004). Because of the rapid modulation observed in the flaring emission, these events likely occur just outside the event horizon and may provide insight into the structure and conditions in the inner accretion regions. Models for the flares have considered various mechanisms for injecting energy into the electrons, including stochastic acceleration, shocks, and magnetic reconnection (e.g., Markoff et al. 2001; Yuan et al. 2004; Liu et al. 2006b). The radiative processes responsible for the flares at each wavelength have also been debated, leaving synchrotron and synchrotron self-Compton (SSC) emission as the most likely candidates for the IR and X-ray emission.

Constraints on the emission processes have improved as more flares have been observed in the IR and X-ray bands. However, a great deal of uncertainty was generated by the conflicting measurements of the IR spectral index during the flares (Ghez et al. 2005; Eisenhauer et al. 2005; Gillessen et al. 2006; Krabbe et al. 2006). In particular, some previous attempts to explain the IR and X-ray spectra with synchrotron and SSC, respectively, have been forced to include complications in order to explain correlated variations of the flux and spectrum (e.g., Liu et al. 2006a; YusefZadeh et al. 2006b; Bittner et al. 2007). In a recent paper, Hornstein et al. (2007; hereafter H07) have used multiband IR observations of several flares to show that, after corrections for stellar contamination, the spectral index of Sgr A* is roughly constant within and between flares, with $S_{\nu} \propto \nu^{-0.6}$. This can be understood as optically thin synchrotron emission from a population of power-law electrons with an $N(E) \propto E^{-2.2}$ energy spectrum. As discussed below, their findings can be used to make a strong case for SSC production of X-ray flares, as has been suggested by many authors. 

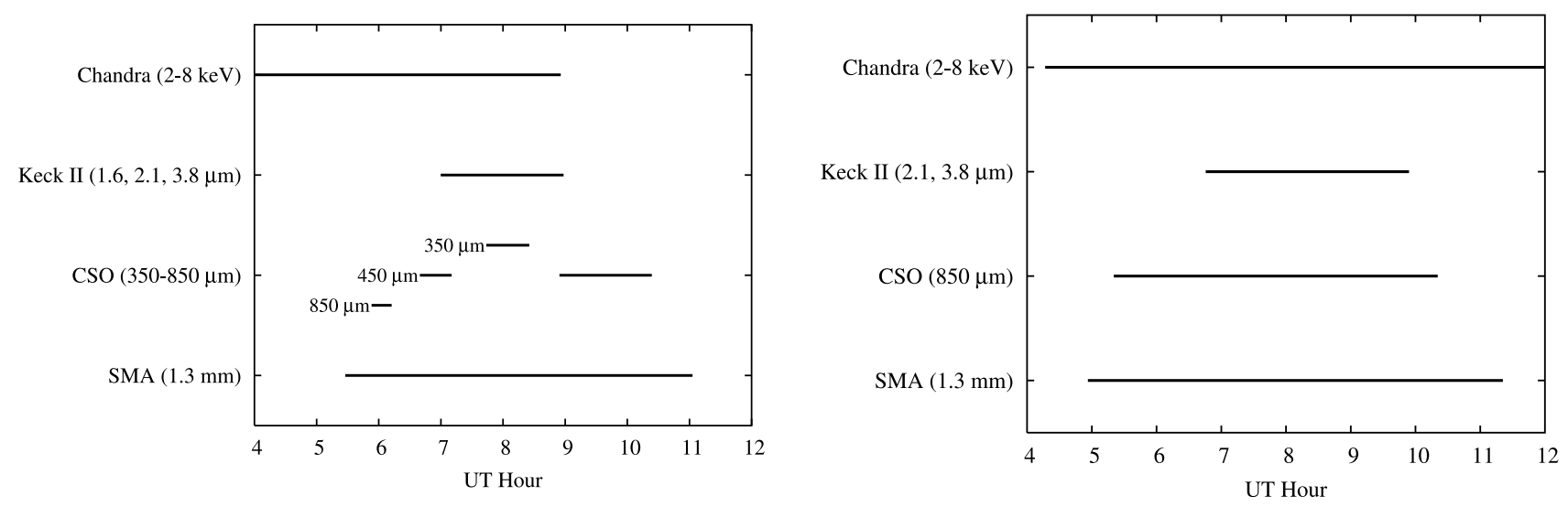

FIG. 1.-Observing windows for the four observatories on 2005 July 31 (left) and 2006 July 17 (right).

Previous considerations of flare emission have largely avoided the temporal evolution of the flares. An exception is the expanding plasmon model used by Yusef-Zadeh et al. (2006a) to explain delays between flares observed in two centimeter-wavelength bands; the extension of this model to shorter wavelengths is discussed in this paper. While understanding the flare creation mechanism (without regard to the flare evolution) is an important goal on its own, much of the potential of the flare measurements to constrain the structure of Sgr A* comes from modeling the flare evolution in time and wavelength as the energized electrons cool and expand through the source. Initially, X-ray and IR flares lacked complementary information at other wavelengths, limiting time- and frequency-domain studies to the information encoded in a single narrow band. To date, a handful of events have been detected simultaneously in X-rays and the IR (Eckart et al. 2004, 2006b; Yusef-Zadeh et al. 2006b), yet because the IR and X-ray flares are observed to be simultaneous the flare evolution has received less attention than the peak spectra.

A few flares have provided evidence for decaying millimeter and submillimeter emission following short-wavelength flares (Zhao et al. 2004; Eckart et al. 2006b; Yusef-Zadeh et al. 2006b). Coordinated observations from centimeter to X-ray wavelengths are now routinely attempted to search for clear flare counterparts across as broad a wavelength range as possible. Here we present the first observations of a flare of Sgr A* detected at submillimeter, IR, and X-ray wavelengths. Using an array of telescopes (§ 2) we are able to measure the amplitude, spectral index, and temporal structure of the flare in each band $(\S 3)$. We also report a second IR/submillimeter flare, detected in the same monitoring campaign, that lacks an X-ray counterpart. We find large delays between the time of the IR and X-ray flares and the submillimeter flares. In $\S 4$ we attempt to constrain the emission processes and dynamics responsible for these and other flares observed in Sgr A*. We find that the timing, spectra, and energetics of the flares imply a synchrotron origin for the IR emission and a SSC X-ray generation mechanism. The decay of these and the submillimeter flares also suggests that nonradiative cooling processes, such as adiabatic expansion, are essential. As an initial step toward understanding the structure of Sgr $\mathrm{A}^{*}$ through the flare changes, we compare the present flare to an existing expansion model. Finally, we use simple scaling arguments to predict the relationship between the IR and X-ray flare luminosities and the delay of the submillimeter counterpart.

Throughout this paper we refer to spectral indices $(\alpha)$ using the convention $S_{\nu} \propto \nu^{\alpha}$. We assume the Reid (1993) distance to
Sgr $\mathrm{A}^{*}, 8 \mathrm{kpc}$, which is consistent with more recent results (e.g., Ghez et al. 2003; Eisenhauer et al. 2003).

\section{OBSERVATIONS AND REDUCTION}

The data presented here were obtained as part of a 2005-2006 campaign to monitor $\mathrm{Sgr} \mathrm{A}^{*}$ simultaneously across a broad range of wavelengths; these results encompass data from four observatories spanning seven decades in wavelength. We report on two strong flares observed at IR and submillimeter wavelengths, only one of which was accompanied by an X-ray flare. The temporal coverage at the various observatories is shown in Figure 1. Details of the individual observations and analysis techniques are discussed in the following sections.

\subsection{X-Ray Data}

The Chandra X-Ray Observatory (Weisskopf et al. 1996) observed the Galactic center on both 2005 July 30/31 and 2006 July 17 using the ACIS imaging array (Garmire et al. 2003). The observations were timed to span the window of Sgr A* visibility from Mauna Kea for coordination with telescopes there. Observational details and analysis procedures followed those of Baganoff et al. $(2001,2003)$. In particular, Sgr A* photometry was obtained from $2-8 \mathrm{keV}$ counts within $1.5^{\prime \prime}$, after subtraction of a background derived from a $2^{\prime \prime}-4^{\prime \prime}$ annulus with point sources and structures excluded.

\subsection{IR Data}

The W. M. Keck II $10 \mathrm{~m}$ telescope observed the Galactic center using the NIRC2 (PI: K. Matthews) near-IR camera and the laser guide star adaptive optics system (Wizinowich et al. 2000; van Dam et al. 2006) on 2006 July 17. Observations were alternately made in the $K^{\prime}\left(\lambda_{0}=2.12, \Delta \lambda=0.35 \mu \mathrm{m}\right)$ and $L^{\prime}$ $\left(\lambda_{0}=3.78, \Delta \lambda=0.70 \mu \mathrm{m}\right)$ photometric bands, with exposure times of 28 and $30 \mathrm{~s}$, respectively, each cycle. The observations span 187 minutes, with 16 minutes of data lost to instrument problems. Additional data were obtained on 2005 July 31 in the $H$ ( $\left.\lambda_{0}=1.63, \Delta \lambda=0.30 \mu \mathrm{m}\right), K^{\prime}$, and $L^{\prime}$ bands, cycling through $22.2,28$, and $30 \mathrm{~s}$ exposures in these bands every 3 minutes. Within the 113 minutes of observations, 9 minutes were lost to telescope problems. The dead time between frames on these nights was typically less than 1 minute. Seeing on both nights was excellent; the resolution achieved at $H$ and $K^{\prime}$ was $62-65$ mas (FWHM) and 80-82 mas at $L^{\prime}$. We refer the reader to $\mathrm{H} 07$ for additional details. 

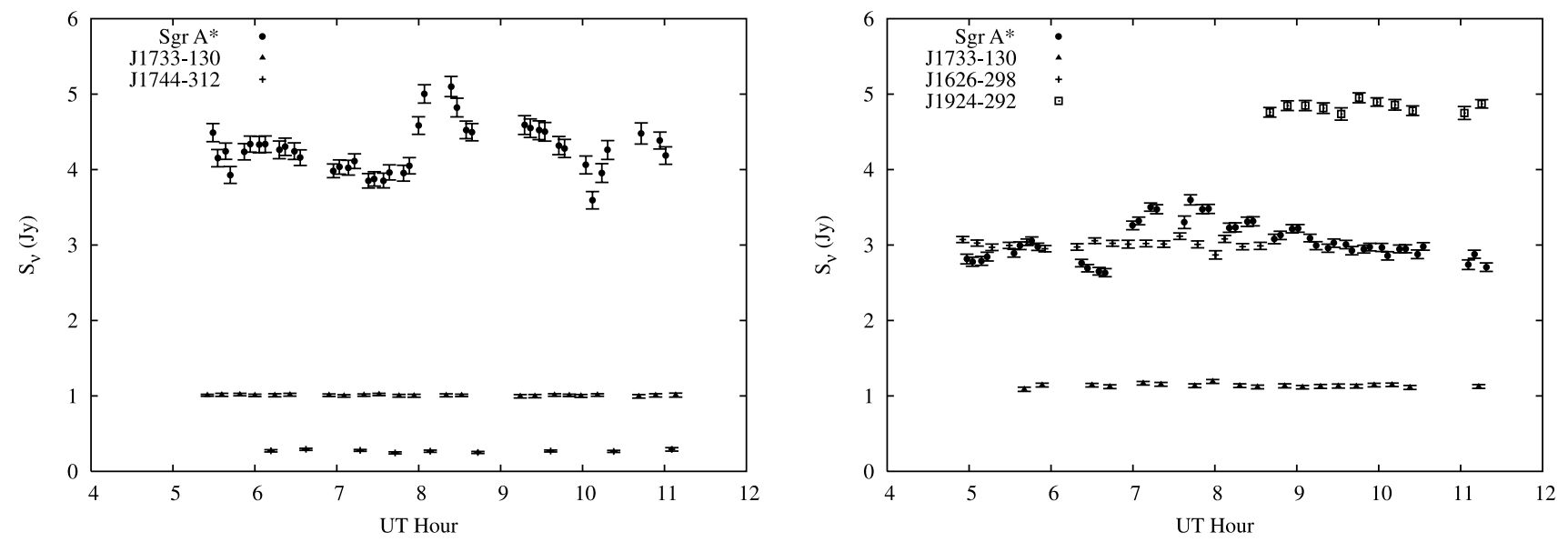

FIG. 2.-Left: SMA light curve from 2005 July 31 . The calibrator was J1733-130, and J1744-312 has been used as a test source to verify the calibration. Right: Light curve from 2006 July 17, with calibrators J1626-298 and J1924-292 and test source J1733-130. [See the electronic edition of the Journal for a color version of this figure.]

\subsection{Submillimeter Data}

The Caltech Submillimeter Observatory (CSO) SHARC-II observation and analysis methods are described by Yusef-Zadeh et al. (2008) with attention to the 2006 July 17 observations at $850 \mu \mathrm{m}$. Observations were also made on 2005 July 31 at 350 , 450 , and $850 \mu \mathrm{m}$, for which the CSO has $8.5^{\prime \prime}, 10^{\prime \prime}$, and $20^{\prime \prime}$ resolution, respectively. For the 2005 observations, $850 \mu \mathrm{m}$ calibration was derived from Callisto (10.3 Jy) and Neptune (27.7 Jy), with an estimated uncertainty of $10 \%$. Confusion caused an additional $\sim 1$ Jy uncertainty in the absolute flux density of Sgr $\mathrm{A}^{*}$. At $450 \mu \mathrm{m}$, Arp 220 (6.3 Jy), Callisto (35 Jy), and Neptune (67 Jy) were used for absolute calibration, with an estimated uncertainty of $25 \%$. Confusion causes an additional $\sim 0.5 \mathrm{Jy}$ uncertainty in the absolute flux density of Sgr $\mathrm{A}^{*}$ at $450 \mu \mathrm{m}$. At $350 \mu \mathrm{m}$, Arp 220 (10 Jy) and Neptune (93 Jy) were used for absolute calibration, with an estimated uncertainty of $25 \%$ and a confusion uncertainty of $\sim 1$ Jy.

Submillimeter Array (SMA) observations of Sgr A* were made on 2005 July 31 and 2006 July 17 (UT). In 2005, seven antennas were used in the SMA "compact north" configuration at $1.32 \mathrm{~mm}$ wavelength $(226.9 \mathrm{GHz})$, covering baseline lengths of 5-53 k $\lambda$ and yielding a synthesized beam of $3.8^{\prime \prime} \times 2.1^{\prime \prime}$ after a $6 \mathrm{hr}$ track. During the track the zenith opacity varied between 0.05 and 0.08 . The SMA polarimetry system (Marrone 2006) was installed for these observations in order to convert the linearly polarized SMA feeds to circular polarization sensitivity. This removes the possibility of confusing linear polarization modulation with total intensity variations. Gain calibration was derived from the quasar $\mathrm{J} 1733-130$, while $\mathrm{J} 1744-312$, just $2.3^{\circ}$ from Sgr $\mathrm{A}^{*}$, was used as a comparison source to verify the calibration. The flux density scale was determined from Uranus, with an uncertainty of $15 \%$. In 2006 , seven antennas were used in the "very extended" configuration, yielding baselines of 27-390 k $\lambda$ and a synthesized beam of $0.6^{\prime \prime} \times 0.5^{\prime \prime}$ after a $6.5 \mathrm{hr}$ track on Sgr A*. The observing wavelength was the same as in 2005 , while the zenith opacity was 0.10 . For these data the polarimetry system was used to make full polarization measurements according to the procedures described in Marrone et al. (2006a). In order to sample all cross-correlations of left and right circular polarization on all baselines, the feed polarizations were modulated in a coordinated pattern with a 4 minute duration; this cycle time set the minimum length of the polarization samples. Instru- mental polarization calibration was obtained through observations of the quasar 3C 279, yielding measurements consistent with those obtained in previous observations at this frequency. As described in Marrone et al. (2007), the calibration precision limits false linear polarization signals to $0.2 \%$. Gain calibration was derived from J1626-298 and J1924-292, with J1733-130 as a verification source. Callisto was used for absolute calibration, with an uncertainty of $15 \%$. For both epochs, the complex calibrator gains were applied to the $\mathrm{Sgr} \mathrm{A}^{*}$ data, and then $\mathrm{Sgr} \mathrm{A}^{*}$ was used for phase-only self-calibration. Projected baselines shorter than $20 \mathrm{k} \lambda$ were excluded from this procedure because of contamination from extended emission around Sgr A*. Flux density measurements were obtained for each time interval (4 minute onsource cycles) by fitting a point source to the calibrated visibilities. Flux density uncertainties were adjusted to account for the precision of the calibrator gain measurements, while the overall flux density scale uncertainties reported above were not included because they should be common to all time intervals. Figure 2 shows the SMA light curves for both epochs, including calibration and verification sources.

\section{RESULTS}

\subsection{Flare Amplitude and Duration}

Figure 3 shows the light curve observed at submillimeter, IR, and X-ray wavelengths on 2006 July 17. All three bands (four telescopes) show a flare between 6 and $8 \mathrm{hr}$ UT. Assuming that the events seen at these wavelengths are related, this is the first flare of Sgr $\mathrm{A}^{*}$ to be observed in all of these bands.

The X-ray flare, centered around 06:10 UT, has a FWHM of 31 minutes and a FWZP of roughly $1 \mathrm{hr}$. At its peak, this flare has a $2-8 \mathrm{keV}$ luminosity of $4.0 \times 10^{34} \mathrm{ergs} \mathrm{s}^{-1}$, approximately 20 times the quiescent X-ray luminosity of Sgr $\mathrm{A}^{*}$. The integrated emission of the flare has a spectral index of $\alpha=0.0_{-1.6}^{+1.0}$ [photon index of $\Gamma=1.0$ for $\left.N(E)=E^{-\Gamma}\right]$, implying a monochromatic luminosity $\left(\nu L_{\nu}\right)$ of $7 L_{\odot}$ at $4 \mathrm{keV}$. Flares of this amplitude or larger have been observed on six occasions in the past (Baganoff et al. 2001, 2002; Goldwurm et al. 2003; Porquet et al. 2003; Bélanger et al. 2005), corresponding to a rate of around $0.6 \pm 0.3$ day $^{-1}$.

The IR observations begin 36 minutes after the peak of the $\mathrm{X}$-ray flare, and Sgr $\mathrm{A}^{*}$ is initially a factor of a few brighter than the minimum emission observed over the night. The 7 (7.5) $\mathrm{mJy}$ peak observed at $K^{\prime}\left(L^{\prime}\right)$ corresponds to $20(12) L_{\odot}\left(\nu L_{\nu}\right)$. Within 


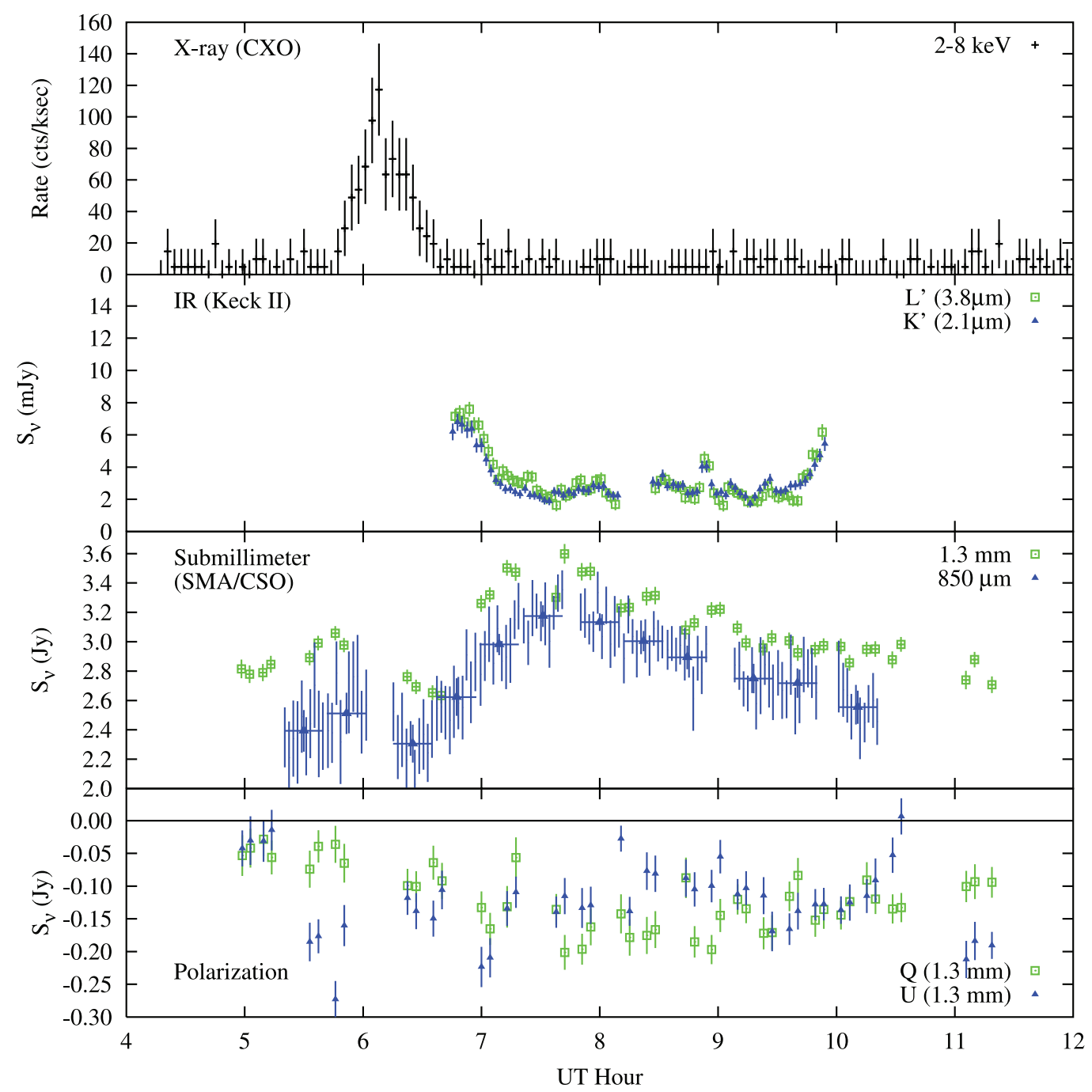

FIG. 3.-Flux density observed during the 2006 July 17 flare in all three bands. For the CSO $850 \mu \mathrm{m}$ data the flux density measurements are shown at their full temporal resolution (vertical bars) and rebinned into 10 minute averages. The zero point of the $850 \mu \mathrm{m}$ flux density scale is uncertain by 1 Jy due to confusion with the surrounding dust emission. The $1.3 \mathrm{~mm}$ polarization measured by the SMA is shown in the bottom panel.

50 minutes, 85 minutes after the X-ray peak, the emission decays to a low level (2-3 mJy). Throughout the IR flare the $K^{\prime}-L^{\prime}$ spectral index is approximately $-0.51 \pm 0.14$, consistent with other IR flares discussed in H07. The spectral index between the $K^{\prime}$ and X-ray peaks is -1.21 , although more negative indices are allowed because the IR peak may have been significantly brighter. Assuming that the $K^{\prime}$ peak was comparable to the largest flares observed to date, $\sim 12 \mathrm{mJy}$, the spectral index would be -1.28 . In previous observations of X-ray flares with IR counterparts, Eckart et al. (2006b) and Yusef-Zadeh et al. (2006b) found $\alpha_{K-\mathrm{X}}=$ -1.12 and $\alpha_{H-\mathrm{X}}=-1.3$, respectively. Assuming an IR spectral index of $-0.6(\mathrm{H} 07)$, the latter is equivalent to $\alpha_{K^{\prime}-\mathrm{X}}=-1.2$.

Although the submillimeter observations span the X-ray and IR flares, there is no submillimeter flare apparent at the time of the maxima in these bands. Prior to the X-ray flare, both telescopes show a small ( $0.2 \mathrm{Jy},<10 \%$ fractional change) rise and fall in flux density. Due to an unfortunate coincidence, neither telescope was observing Sgr $\mathrm{A}^{*}$ precisely at the peak of the X-ray flare, but there is no suggestion of a missed increase in emission from the data immediately before or after the gap. However, a large (1 Jy) flare is seen at both wavelengths, peaking more than an hour after the $\mathrm{X}$-ray flare. At $1.3 \mathrm{~mm}$ and $850 \mu \mathrm{m}$ the monochromatic luminos- ities of 1 Jy flares are 4.6 and $7.0 L_{\odot}$, respectively. Events of this magnitude have been seen in previous observations at $1.3 \mathrm{~mm}$ and $850 \mu \mathrm{m}$ (e.g., Marrone et al. 2006a; Eckart et al. 2006b; YusefZadeh et al. 2006b); they occur with a frequency of $\sim 1.2$ day $^{-1}$ based on 20 epochs since 2004. The decay of this flare is well approximated at both wavelengths by an exponential with a time constant of $2 \mathrm{hr}$. A similar decay was also suggested by the $850 \mu \mathrm{m}$ data presented by Eckart et al. (2006b). The spectral index of the flaring component is tough to determine because of the absolute calibration uncertainty and the difficulty in determining the nonflaring flux. Assuming that the minimum flux density observed at each wavelength represents the stable component, the submillimeter spectral index during the flare rise (07:00-07:30 UT) is $\alpha_{\text {submm }}=-0.1 \pm 0.2 \pm 0.4$, with a mean of $0.4 \pm 0.1 \pm 0.4$ after the flare peak. For each spectral index we separate the errors resulting from the measurement error (first) from the constant error due to uncertainty in the absolute calibration of the two observations (second). The change in spectral index across the peak of the flare is an increase of $0.5 \pm 0.2$.

On 2005 July 31 we also observed a strong IR flare, among the brightest yet detected (Fig. 4). It was accompanied by a $1.3 \mathrm{~mm}$ flare of similar amplitude to that of 2006 July 17 . The IR and 


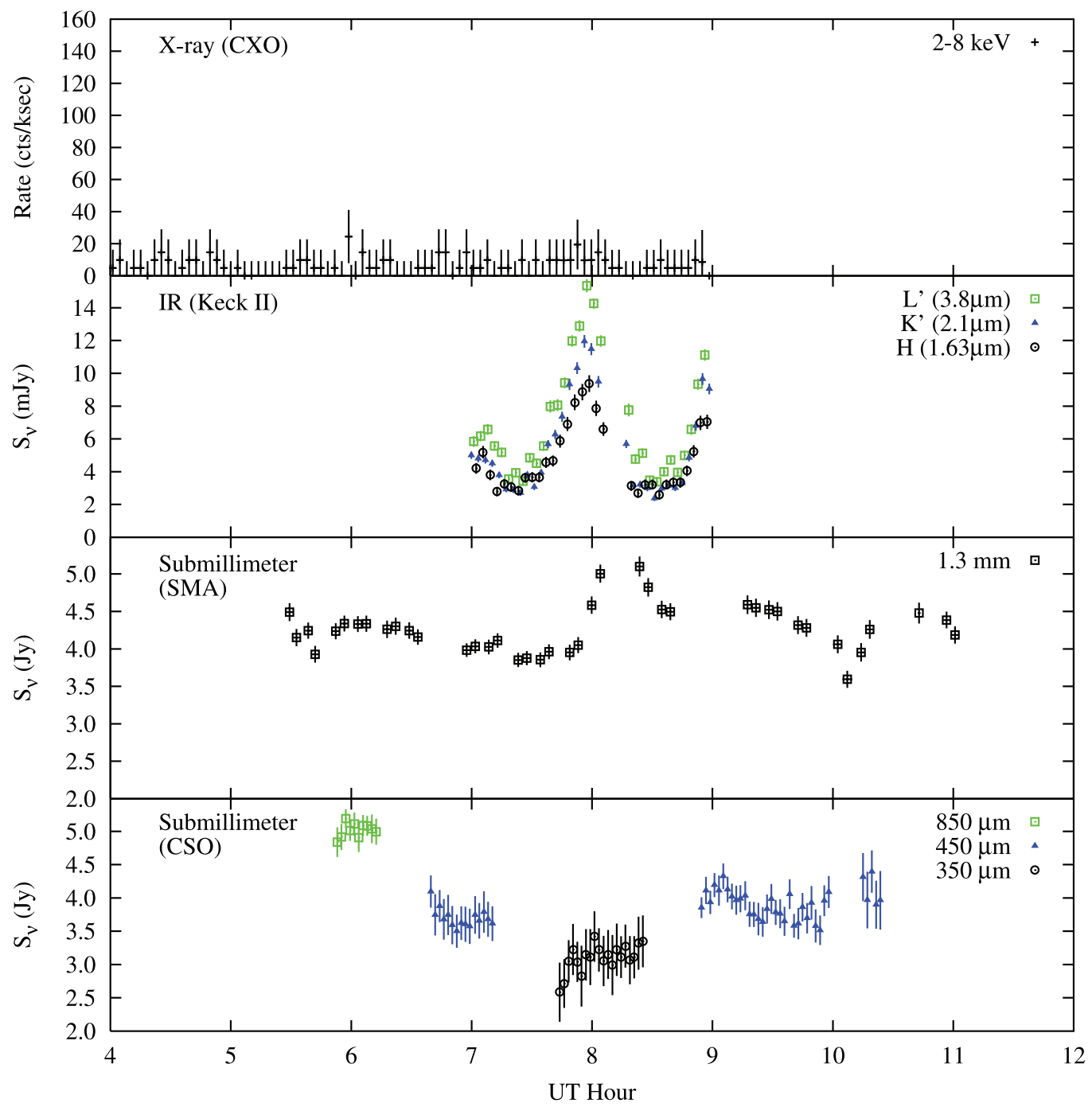

FIG. 4.-Flux density observed on 2005 July 31 in all three bands. The CSO data (bottom) were obtained in three different filters, and the frequent switching between wavelengths makes correlation analysis difficult, although the $450 \mu \mathrm{m}$ data generally follow the trend observed at $1.3 \mathrm{~mm}$ by the SMA (second from bottom). These have been separated for clarity and plotted to the same scale. The zero point of the flux density scales at 850,450 , and $350 \mu \mathrm{m}$ are uncertain by $1,0.5$, and $1 \mathrm{Jy}$, respectively, due to confusion. No flare is detected in the X-ray observations during this interval, as reported in H07. The X-ray and IR data are plotted on the same scale as Fig. 3.

submillimeter-to-IR indices are very similar to those in the 2006 flare; $\mathrm{H} 07$ report a spectral index of $-0.62 \pm 0.21$ between $K^{\prime}$ and $L^{\prime}$ and $\alpha_{1.3 \mathrm{~mm}-K^{\prime}} \simeq-0.7$ in both epochs. As noted by $\mathrm{H} 07$, there is no appreciable change in X-ray flux during these observations, despite coverage beginning more than $10 \mathrm{hr}$ before the start of the Keck IR data. The nondetection of X-ray emission places an upper limit of $\alpha_{K^{\prime}-\mathrm{X}}<-1.50$.

\subsection{Correlation Analysis}

The peak of the 2006 July 17 X-ray flare occurred before the beginning of our IR observations. The probability of the IR and $\mathrm{X}$-ray flares coinciding by chance within this time interval is nonnegligible given the observed IR flare rate, as discussed by H07. However, all previous X-ray flares that have occurred during IR observations have been accompanied by an IR flare, with no measurable time delay between the two wavelength bands ( $\leq 10$ minutes; Eckart et al. 2006b, 2008; Yusef-Zadeh et al. 2006b). The apparent flare peak at the beginning of the IR observations is consistent with substructure observed in previous IR events (e.g., Eckart et al. 2006b). We therefore assume that the IR and X-ray peaks are coincident and expect that the maximum IR flux density was greater than the $\sim 7 \mathrm{mJy}$ at the start of these observations. We refer to $\mathrm{H} 07$ for further discussion.

Neither of the flare events in 2005 and 2006, as marked by the IR and X-ray emission, shows coincident submillimeter activity. Both, however, show submillimeter flares of unusual amplitude after the X-ray or IR emission peak. The apparent delay between the submillimeter and IR/X-ray flares makes the assertion of a relationship between these events even more uncertain than the IR/X-ray connection described above, but circumstantial evidence of a relationship is building. From campaigns between 2004 and 2006 there are approximately $52 \mathrm{hr}$ of joint X-ray/submillimeter observations of Sgr A* yielding just one X-ray flare, the 2006 flare presented here (Eckart et al. 2006b; Yusef-Zadeh et al. 2006b; F. K. Baganoff et al., in preparation). A 2004 flare that occurred $2.3 \mathrm{hr}$ before the start of the submillimeter observations was also followed by a $0.8 \mathrm{Jy}$ decline in $870 \mu \mathrm{m}$ flux over the first $2 \mathrm{hr}$ of the submillimeter light curve (Eckart et al. 2006b). A similar number of hours of simultaneous IR/submillimeter measurements (Eckart et al. 2006b; Yusef-Zadeh et al. 2006b; this work) have produced three instances of IR flares followed by submillimeter flares. In the case of the 2005 flare presented here and the 2004 


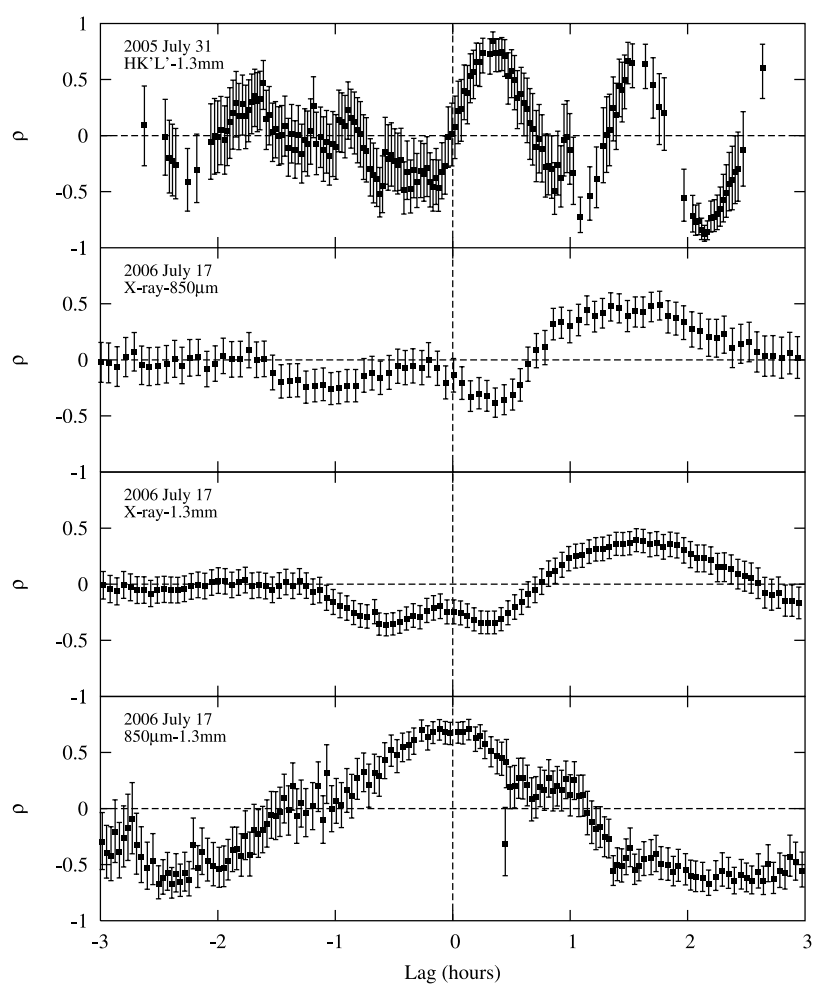

FIG. 5.-ZDCFs for the available light curves for the 2005 and 2006 flares. Only observations that span the apparent flare peak in each band have been crosscorrelated. The top panel shows the cross-correlation of the $1.3 \mathrm{~mm}$ data (Fig. 4, second from bottom) with the spectral-average IR light curve. The latter is the combination of the $H, K^{\prime}$, and $L^{\prime}$ light curves with the first two scaled by the $\alpha=$ -0.62 mean spectral index to the $L^{\prime}$ flux density scale to generate a single light curve with superior sampling. The bottom three panels show the three crosscorrelations of the $2006 \mathrm{X}$-ray, $850 \mu \mathrm{m}$, and $1.3 \mathrm{~mm}$ data in Fig. 3. A positive lag indicates that structure in the second data set appears after that in the first.

September 4 flare in Yusef-Zadeh et al. (2006b), the submillimeter event occurs after a large IR flare but precedes a smaller flare. In these cases it is not clear which IR event to associate with the submillimeter, if any, but we note that in all five of these cases the submillimeter flare follows the largest event observed at the shorter wavelengths. If the X-ray/IR events are unrelated to the submillimeter we would expect an equal number of flares before and after the X-ray/IR flares. We therefore proceed on the assumption that the two submillimeter flares presented here are related to the X-ray/IR flares.

In Figure 5 we show the cross-correlation of the 2006 submillimeter and X-ray light curves. We have employed the $z$-transform discrete correlation function (ZDCF) analysis of Alexander (1997) in order to treat properly the irregular sampling of these data sets. We find no significant delay between the $1.3 \mathrm{~mm}$ and $850 \mu \mathrm{m}$ light curves, with the $850 \mu \mathrm{m}$ peak leading by $2 \pm 12$ minutes. Cross-correlation with the X-ray light curve indicates delays of $96 \pm 14$ and $97 \pm 17$ minutes for the $850 \mu \mathrm{m}$ and $1.3 \mathrm{~mm}$ data. The cross-correlation of the $2005 \mathrm{IR}$ and $1.3 \mathrm{~mm}$ data is also shown in Figure 5 (top), where the IR flux is represented by the spectralaverage light curve, obtained by scaling the $H$ and $K^{\prime}$ flux densities to the $L^{\prime}$ band through the factor $\left(\nu / \nu_{L^{\prime}}\right)^{\alpha}$, where $\alpha=-0.62$, the mean $K^{\prime}-L^{\prime}$ spectral index of the flare (H07). This composite light curve leads the $1.3 \mathrm{~mm}$ flare by $20 \pm 5$ minutes; crosscorrelation with each individual IR light curve yields similar results, and the intercorrelations of the IR light curves show no evi- dence for relative delays. The second peak in the cross-correlation is spurious, arising from the chance alignment of the gap in the SMA data with a minimum in the IR light curve.

The lag between the submillimeter and X-ray flares in the 2006 event is nearly 80 minutes longer than the submillimeter-IR lag in the 2005 flare. However, because the 2005 flare shows no $\mathrm{X}$-ray emission and we lack IR coverage at the expected peak of the 2006 flare, we cannot compare cross-correlations of the same pair of wavelengths between the two flares. If the plateau at the beginning of the 2006 IR data truly represents the peak of the IR flare, the delay between IR and submillimeter would be $40-$ 45 minutes shorter, although still measurably longer than that observed in 2005 .

\subsection{Flare Polarization}

Although linear polarization has been detected in $\mathrm{Sgr} \mathrm{A}^{*}$ at submillimeter and near-IR wavelengths (e.g., Aitken et al. 2000; Bower et al. 2003; Marrone et al. 2006a; Eckart et al. 2006a), of the observations presented here only the 2006 July 17 SMA observations were designed to measure polarization. The $1.3 \mathrm{~mm}$ polarization light curve for that epoch is shown in Figure 3 (bottom). The fractional polarization varies from $1 \%-2 \%$ at the start of the track to as much as $8 \%-9 \%$. The polarization position angle varies between $90^{\circ}$ and $130^{\circ}$ in the 4 minute samples.

If the submillimeter flare emission arises from the synchrotron process, the flare might be expected to be highly polarized. To examine the flare polarization, we rebin the data in half-hour intervals (typically, four 4 minute observing cycles) and subtract the total intensity $\left(I_{0}\right)$ and polarization $\left(Q_{0}, U_{0}\right)$ averaged over the four samples that precede the onset of the submillimeter flare. Although Sgr A* often shows dramatic polarization modulation (magnitude and direction) even during periods of quiescence (Marrone 2006), making the assumption of a single $Q_{0}$ and $U_{0}$ possibly unreliable, the resulting background-subtracted light curve (Fig. 6) reveals interesting changes. As the excess Stokes $I$ rises and falls, a polarization component $\left(P_{\text {excess }}\right)$ also appears and fades, suggesting that the flare emission is significantly polarized. Previous IR and centimeter-wave observations of Sgr A* have also shown evidence of polarized flare emission (Eckart et al. 2006a; Meyer et al. 2006b; Trippe et al. 2007; Yusef-Zadeh et al. 2007). The polarization fraction of the excess emission $\left(m_{\text {excess }}\right)$ is observed to increase from $9.4 \% \pm 1.9 \%$ while the flare intensity is increasing (the first two bins after the flare onset) to a weighted average of $16.5 \% \pm 2.3 \%$ after the peak (exclusion of the last bin causes an insignificant change in this average). This increase is consistent with a synchrotron flare that is evolving from optically thick to optically thin, assuming a power-law electron distribution with $N(E) \propto E^{-p}$ and $p>-0.45$. For the electron index indicated by the constant IR spectral index, $p=$ 2.2 , the polarization fraction would be expected to change from $11 \%$ to $71 \%$ through this transition if the flaring region lacked any appreciable random magnetic field component. The smaller change observed here suggests that there is significant disorder in the field revealed as the flare becomes optically thin, or substantial internal Faraday rotation at $1.3 \mathrm{~mm}$.

The variation of the excess $Q$ and $U$ through the flare represents a rotation of the polarization; the total excess polarization (Fig. 6, bottom panel, circles) remains nearly constant. Comparing the data point on the rising edge of the flare with the six after the peak, we find that the polarization angle changes by $40^{\circ}$, not as large as the expected $90^{\circ}$ change through a transition from optically thick to thin synchrotron emission. However, the magnitude of this change depends strongly on the choice of $Q_{0}$ and 


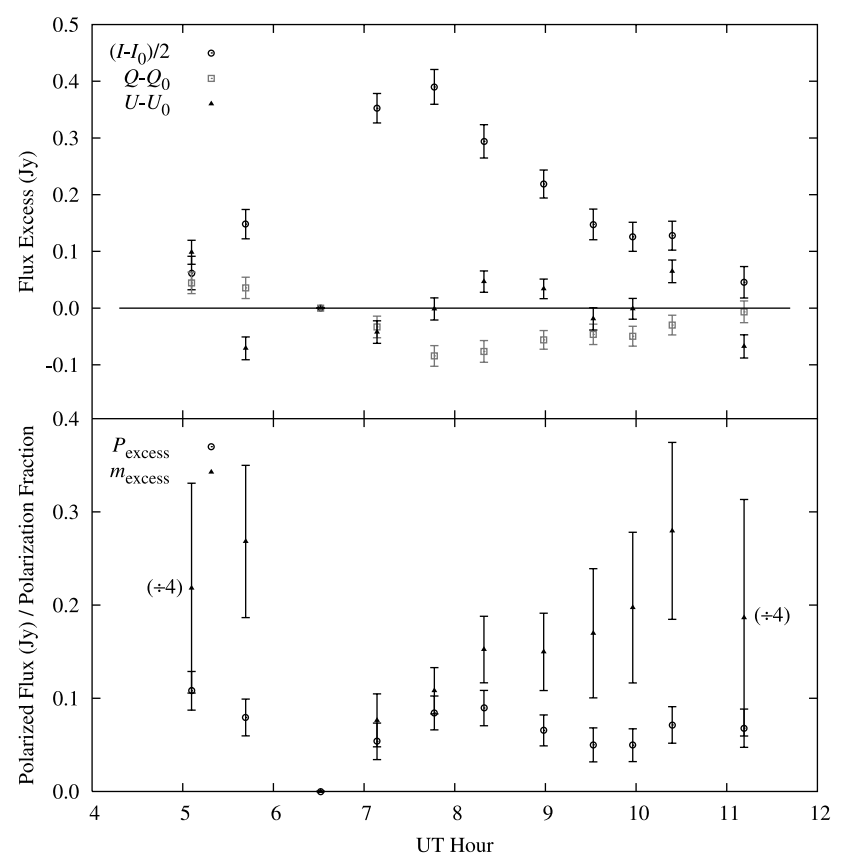

FIG. 6.-Polarization changes during the 2006 July 17 flare at $1.3 \mathrm{~mm}$. The data are binned in half-hour intervals (3-6 samples). Top: Stokes intensities after subtracting the values in the $630 \mathrm{UT}$ bin $\left(I_{0}=2.98 \mathrm{Jy}, Q_{0}=-89 \mathrm{mJy}, U_{0}=\right.$ $-128 \mathrm{mJy}$ ). The remaining emission is ascribed to the flare (the two points that precede the flare are also shown). Bottom: Polarized emission in the flare. Excess polarization is calculated as $P_{\text {excess }}=\left[\left(Q-Q_{0}\right)^{2}+\left(U-U_{0}\right)^{2}\right]^{1 / 2}$, and the polarization fraction ( $\left.m_{\text {excess }}\right)$ is the ratio of $P_{\text {excess }}$ and $I-I_{0}$. The first and last points have large and uncertain $m_{\text {excess }}$; these points and their errors have been scaled by one-fourth. [See the electronic edition of the Journal for a color version of this figure.]

$U_{0}$ and could be made to agree with the prediction if these quantities are slightly more negative than assumed.

\section{DISCUSSION}

\subsection{Emission Mechanisms and Electron Cooling}

After several years of coordinated multiwavelength monitoring of Sgr $\mathrm{A}^{*}$, the physical conditions and mechanisms responsible for its flaring are becoming clear. The IR observations of Sgr $\mathrm{A}^{*}$ in flaring and quiescent states by $\mathrm{H} 07$ show a consistent $\alpha_{\mathrm{IR}}=-0.6$ spectrum, independent of the instantaneous flux density and its derivative. The spectral index suggests that the IR photons are optically thin synchrotron emission from power-law electrons $\left[N(E) \propto E^{-p}\right]$ with $p=(1-2 \alpha)=2.2$. Moreover, the stability of the spectral index as the flares decay is inconsistent with the $\Delta \alpha \geq-1 / 2$ change expected if the decay results from radiative cooling of the electrons (Pacholczyk 1970). The electron cooling timescale due to synchrotron losses is (e.g., Krolik 1999)

$$
t_{\mathrm{cool}}=1.3 \times 10^{12} \nu^{-1 / 2} B^{-3 / 2} \mathrm{~s},
$$

where the frequency $(\nu)$ is in $\mathrm{Hz}$ and the magnetic field $(B)$ is in G. Assuming that after the flare peak the IR-emitting electrons are no longer produced in large numbers and can no longer hide a change in spectrum, the 25 minute decay of the IR flares limits the field in the emission region to $\sim 20 \mathrm{G}$. At this field strength, electrons emitting at $K^{\prime}$ have a Lorentz factor of $\gamma \geq 1600$.

Measurements of bright radio and submillimeter flares also imply that we are observing synchrotron flares that decay due to nonradiative electron cooling. The strongly polarized flare emission shown in Figure 3 is suggestive of a synchrotron origin. In both of the submillimeter flares considered here the excess flux fades within $2 \mathrm{hr}$, much more quickly than could be explained by synchrotron losses. Equation (1) predicts that submillimeteremitting electrons should cool 20 times more slowly than those observed in the IR bands, very different from the observed factor of a few difference in decay time. Similarly rapid decay has been observed in flares at lower frequencies (Yusef-Zadeh et al. 2006a). The behavior of the long-wavelength flares and the achromaticity of the IR decay imply that an energy-independent process, such as expansion, dominates the energy loss. Magnetic flux-conserving expansion also reduces the magnetic field and therefore could allow a somewhat higher initial $B$ and smaller Lorentz factor than those quoted above.

Between 2000 and 2006, Chandra and XMM-Newton have found 11 significant increases in the X-ray luminosity of Sgr A* (Baganoff et al. 2001, 2002; Goldwurm et al. 2003; Porquet et al. 2003; Eckart et al. 2004, 2006b; Bélanger et al. 2005; this work). Typically, these flares last for $0.5-2 \mathrm{hr}$, much longer than the synchrotron lifetime for reasonable estimates of the magnetic field strength, so production of the X-ray flares through direct synchrotron emission would require sustained injection of high-energy electrons throughout the flare (e.g., Baganoff et al. 2001; Markoff et al. 2001). On every occasion where IR data have been available, IR counterparts to these flares have been observed (Eckart et al. 2004, 2006b; Yusef-Zadeh et al. 2006b; this work). For the two flares with the best data, those where the flare rise and fall was observed in both bands, there is no significant delay between the two wavelengths (Eckart et al. 2006b; Yusef-Zadeh et al. 2006b). $\mathrm{H} 07$ also noted the correspondence between the X-ray spectral indices $\left(\alpha_{\mathrm{X}}\right)$ and their mean $\alpha_{\mathrm{IR}}$ for all but the brightest X-ray flare observed to date. Finally, as discussed in $\S 3.1$, the spectral index between IR and X-ray wavelengths is variable $\left(\alpha_{K-X}\right.$ ranges from -1.1 to $<-1.5)$ but is reliably more negative than the spectral indices within the IR or X-ray bands. When taken together, these points demonstrate that the X-rays are produced through inverse-Compton scattering of the lower frequency spectrum (see also H07; Yusef-Zadeh et al. 2006b).

\subsection{Expanding Plasmon Flare Evolution Model}

Yusef-Zadeh et al. (2006a) proposed that the temporal and spectral behavior of centimeter-wavelength flares in Sgr A* could be explained in an expanding synchrotron plasmon picture (Shklovskii 1960; Pauliny-Toth \& Kellermann 1966), following the formulation of van der Laan (1966). Eckart et al. (2006b) also proposed an expansion model, although with a less direct connection to previous work. Fundamental to this model is the adiabatic cooling of electrons in the plasmon and the flux-conserving diminution of the magnetic field, which provide the nonradiative decreases in synchrotron output that we require. The model predicts smaller and later flare peaks at longer wavelengths, with the spectral indices characteristic of, respectively, optically thick and thin synchrotron $[\alpha=2.5$ and $(1-p) / 2]$ before and after the flare peak at a given wavelength.

This particular model can be tested in new ways with the 2006 flare because we have observed the flare at two optically thick wavelengths $(1.3 \mathrm{~mm}$ and $850 \mu \mathrm{m})$, know the electron spectral index from the IR observations $(p=2.2)$, and from the X-ray data can pinpoint the time at which the putative expansion was initiated. We found above that the submillimeter spectral index prior to the flare peak is $-0.1 \pm 0.5$ in the flaring component, inconsistent with the expected value of 2.5. This latter number is a direct result of the assumption of a homogeneous plasmon, but 
allowing variations in the electron density and magnetic field with optical depth, as in a jet or other inhomogeneous structure (e.g., de Bruyn 1976), is well known to produce arbitrary spectral shapes. Similarly, the optically thin spectral index was found to be $0.4 \pm 0.5$, just marginally consistent with the -0.6 expected from the electron spectrum. Dent (1968) pointed out that the lightcurve maxima at two wavelengths satisfy $S_{m, 1} / S_{m, 2}=\left(\nu_{1} / \nu_{2}\right)^{\delta}$, where $\delta=(7 p+3) /(4 p+6)$ (ranging from 1 to 1.46 for $p=$ $1-5)$. For our submillimeter data and $p=2.2$, we expect the $850 \mu \mathrm{m}$ peak to be 1.7 times brighter than the $1.3 \mathrm{~mm}$ peak. We instead find the amplitude of the flares in these two bands to be very similar, $\left(S_{850 \mu \mathrm{m}} / S_{1.3 \mathrm{~mm}}\right)=1.15 \pm 0.15$, consistent with $p \sim 0$. Finally, within this expansion model the relative timing of the flare peaks at these two wavelengths is $\left(t_{1} / t_{2}\right)=\left(\nu_{1} / \nu_{2}\right)^{\epsilon}$, $\epsilon=-(p+4) / \beta(4 p+6)$, for expansion as $r \sim t^{\beta}$. Here the flare peaks at short wavelengths (IR/X-ray) at $t=t_{0}$, the scale time, measurable at some optically thick wavelength as $t_{0}=3 S / \dot{S}$ (van der Laan 1966). Derivation of the scale time is quite uncertain due to the quiescent emission and short rise time, but from the $850 \mu \mathrm{m}$ light curve we infer $t_{1.3 \mathrm{~mm}}-t_{850 \mu \mathrm{m}}=34$ minutes, and setting $t_{0}=0$ places a lower limit of 18 minutes on the expected delay for linear expansion $(\beta=1)$. The latter is marginally inconsistent with the observed delay, while the former is discrepant at $3 \sigma$. We also note that in this model the minimum delay between $7 \mathrm{~mm}$ and $850 \mu \mathrm{m}$ should be 135 minutes, so in this context we do not expect any relation between flares observed at 7 and $14 \mathrm{~mm}$ in Yusef-Zadeh et al. (2008) and the large $1.3 \mathrm{~mm} / 850 \mu \mathrm{m}$ flare considered here.

Perhaps a more important problem is revealed by considering the expansion rate expected for the relativistic plasmon. Although Yusef-Zadeh et al. (2006a) invoke an expansion speed of $0.02 c$, the sound speed near the black hole should approach the relativistic limit of $c / \sqrt{3}$. In the case of the 2006 flare, the submillimeter peaks occur nearly 100 minutes after the event that initiated the putative expansion, implying an expansion distance of $10^{14} \mathrm{~cm}$ $\left(100 r_{\mathrm{S}}\right)$. Although the expansion speed may decrease as the plasmon entrains material, this estimate is nearly 2 orders of magnitude larger than the likely size of Sgr $\mathrm{A}^{*}$ at submillimeter wavelengths. Extrapolations of millimeter-wavelength VLBI measurements (Bower et al. 2004, 2006; Shen et al. 2005) suggest an intrinsic quiescent source size of $\sim 2 r_{\mathrm{S}}$ at $850 \mu \mathrm{m}$. Further evidence for the small submillimeter size comes from SED measurements that find the turnover in the submillimeter spectrum expected from the transition to optically thin emission (Marrone et al. 2006b; Marrone 2006). Therefore, $25 \%$ of the luminosity of Sgr A* near $1 \mathrm{~mm}$ would need to be produced by a plasmon that has roughly $50^{2}$ times the surface area of the quiescent source, implying a remarkably low brightness temperature in the plasmon.

The expanded size can be transformed back to an initial size through the opacity law. Under the assumptions of van der Laan (1966), the synchrotron opacity depends on the frequency and expanded size as

$$
\left(\frac{\tau}{\tau_{0}}\right)=\left(\frac{\lambda}{\lambda_{0}}\right)^{(p+4) / 2}\left(\frac{R}{R_{0}}\right)^{-(2 p+3)}
$$

where $\tau_{0}$ is the opacity at a reference wavelength $\lambda_{0}$ and $\left(R / R_{0}\right)$ is the expansion factor. Using $p=2.2$, assuming that initially $\tau_{3.8 \mu \mathrm{m}}<0.5$ to match the spectral index stability constraint (H07) and that at the time of the $850 \mu \mathrm{m}$ peak $\tau=1.6$ as predicted by the model for this electron spectrum (Yusef-Zadeh et al. 2006a), equation (2) shows that $\left(R / R_{0}\right)<8.3$. If the plasmon expands by no more than this factor before reaching a size of $100 r_{\mathrm{S}}$ at the time of the $850 \mu \mathrm{m}$ peak, the initial size is at least $12 r_{\mathrm{S}}$. This source size would dramatically overproduce the observed IR luminosity unless the density were very low $\left(n_{e} \sim 10^{4} \mathrm{~cm}^{-3}\right)$ or the assumption of homogeneity were removed.

\subsection{Other Dynamic Flare Models}

It is clear that although the van der Laan (1966) plasmon model grossly predicts some features observed in this flare, it is inadequate to describe the data presented here. However, this model is just one realization of a family of models that describe the scaling of energy and the magnetic field under expansion. The physics and geometry/dimensionality of the expansion may prescribe other scaling relations (e.g., Königl 1981) or more complicated variations (e.g., Falcke \& Markoff 2000). It is established above that the properties of Sgr $\mathrm{A}^{*}$ flares require nonradiative electron cooling ( $\S 4.1)$. Detailed models that describe the density and field structure in the accretion flow or outflow can also predict the evolution of an expanding region, so time-resolved multiband flare observations can directly test the structure of these models.

It has often been argued that the submillimeter spectrum of Sgr A* is dominated by an electron component that is not significant at other wavelengths, the "submillimeter bump" (Melia \& Falcke 2001). Our SMA and CSO observations fall on the longwavelength side of the peak of this bump, where the synchrotron emission from this component is optically thick. It is therefore possible for the properties of the submillimeter flare to be significantly altered by the excess opacity. For example, for some period of time the ambient submillimeter bump electron population, often taken to be thermal (Yuan et al. 2003), could enshroud otherwise observable emission from the flaring region. However, the submillimeter photosphere is believed to be small (few $r_{\mathrm{S}}$ ) based on extrapolated VLBI size measurements, and it therefore seems unlikely that this mechanism can hide an expanding blob for long. It is also possible that the flare electrons that produce submillimeter radiation are not injected into a power-law tail but instead are heated into a thermal spectrum. In this case, the differing dependence of the thermal synchrotron absorption coefficient on the source properties will change the simple relationship between opacity and expansion derived for power-law electron distributions. We have made no attempt to treat these possibilities, although they are likely to be very important for proper modeling of flares with submillimeter observations.

Observations of repeated structures in IR and X-ray flares (e.g., Eckart et al. 2006a; Meyer et al. 2006b; Bélanger et al. 2006) have often been attributed to plasma "hot spots" orbiting the black hole (e.g., Broderick \& Loeb 2006; Marrone et al. 2006b; Meyer et al. 2006a; Trippe et al. 2007). In these interpretations, intensity and polarization features with $\sim 20$ minute cycle times are ascribed to orbital motion, with several cycles observed in some flares. If these features are to persist for multiple orbits they must not expand significantly. However, the decay timescales for X-ray flares, indicative of expansion, are typically comparable to a single orbital period and conflict with the required plasmon confinement. Unless separate mechanisms are invoked for the "periodic" single-band flares and multiwavelength flares shown here and elsewhere, it is unlikely that such hot spots survive for several orbits.

\subsection{X-Ray Emission and the Submillimeter Delay}

There are two striking differences between the 2005 and 2006 flares in Figures 3 and 4. First, although the 2005 IR flare reaches twice the peak (observed) flux of the 2006 flare, it shows no measurable X-ray emission above that from the quiescent extended 


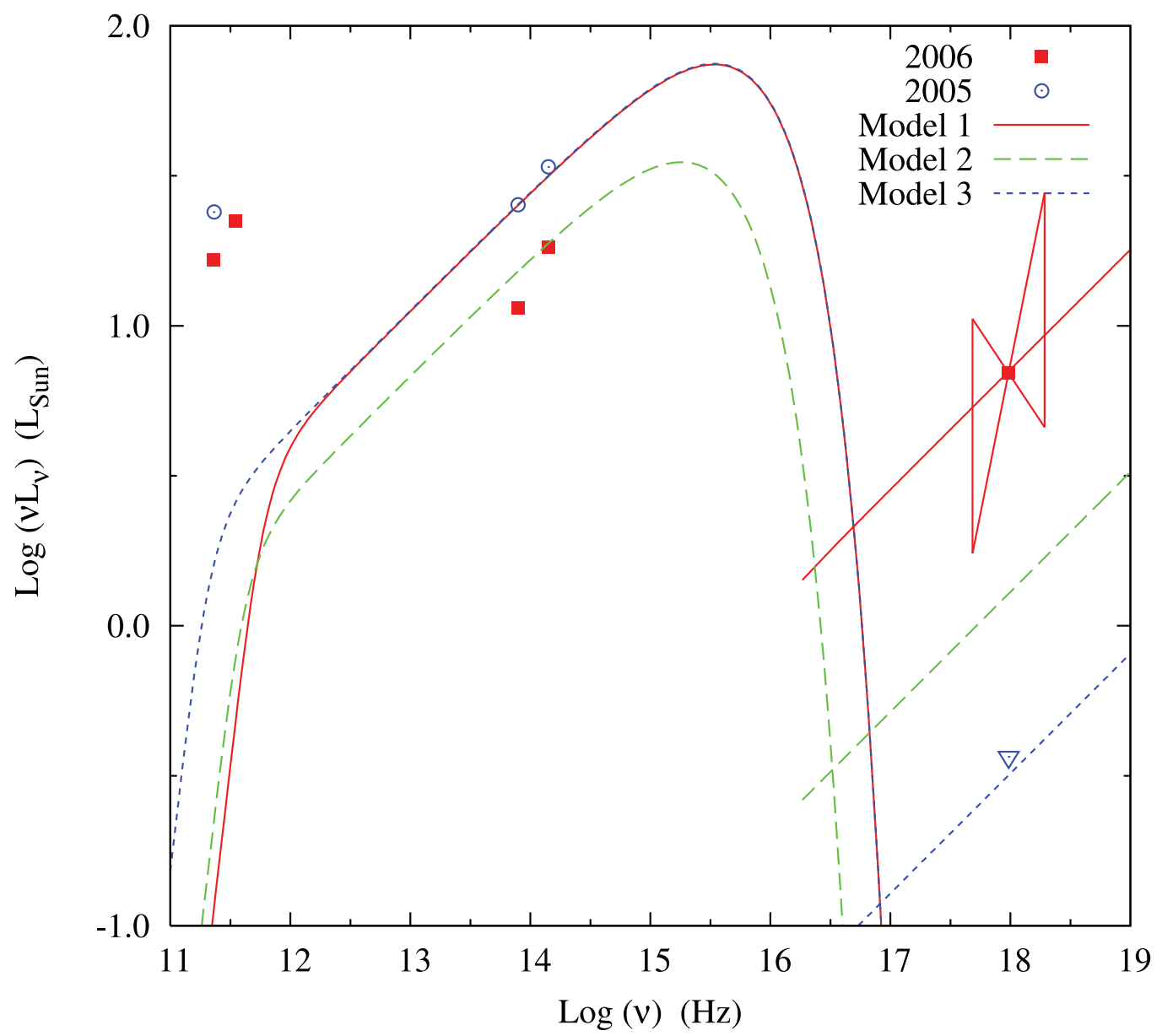

FIG. 7.-SED of the peak emission in the 2005 and 2006 flares. The X-ray emission for the 2005 flare is shown as an upper limit at 4 keV (triangle). Also shown are three synchrotron-SSC models for the IR and X-ray emission from the flares. The 2006 X-ray flare and presumed peak IR emission (chosen to match the 2005 flare maxima) are well fit by model 1 (solid line), while model 2 (long-dashed line) approximates the 2006 flare at the start of the IR coverage. Model 3 (short-dashed line) matches the 2005 IR flare and falls below the X-ray upper limit. Model parameters are given in Table 1 and discussed in $\S 4.4$. The submillimeter peaks are not fit by these models because the bulk of the submillimeter photons and low-energy electrons, observed as the quiescent emission, are not accounted for in the flare model, and because these peaks occur long after the IR and X-ray peaks.

component. Second, the delay between the short-wavelength and submillimeter flares in 2005 is much shorter than in 2006, although we cannot rule out that the submillimeter flare is related to the IR flare seen around 7 UT rather than the much stronger flare at 8 UT. Presuming that the X-rays arise from inverseCompton processing of the longer wavelength spectrum and that the late appearance of the submillimeter emission results from optical depth changes, we use a simple synchrotron-SSC source model to estimate how the X-ray/IR ratio and submillimeter delay should be related.

We begin with a homogeneous spherical synchrotron source of radius $R$, electron density $n_{e}$, and magnetic field $B$. We assume a power-law distribution of electrons between $\gamma_{\min }$ and $\gamma_{\max }$, $N(\gamma) \propto \gamma^{-p}$, with $p=2.2$ as determined from the IR spectrum. The scaling of the synchrotron and SSC spectra of such a source were described by Bloom \& Marscher (1996), and we follow their analysis here. Approximating the spectrum of a single electron of energy $\gamma$ by a delta function at the characteristic emission frequency $\nu_{\gamma}=2.8 \gamma^{2} \mathrm{BMHz}$, the synchrotron spectrum $S_{\nu}^{S}$ of the source at optically thin frequencies is then proportional to

$$
S_{\nu}^{S} \propto n_{e} R^{3} B^{(1+p) / 2} \nu^{(1-p) / 2}
$$

The SSC spectrum $\left(S_{\nu}^{\mathrm{IC}}\right)$ is proportional to the Thomson optical depth of the sphere (roughly $n_{e} R \sigma_{\mathrm{T}}$ ) times $S_{\nu}^{S}$,

$$
S_{\nu}^{\mathrm{IC}} \propto n_{e}^{2} R^{4} B^{(1+p) / 2} \nu^{(1-p) / 2} .
$$

The ratio of these two equations, namely, $S^{\mathrm{IC}} / S^{S} \propto n_{e} R$, provides an explanation for the variation in $\alpha_{\mathrm{IR}-\mathrm{X}}$ noted in $\S 3.1$ : differences in the density and size of the flaring region from flare to flare. This model preserves the spectral similarity of the X-ray and IR flares, matching the observations.

We can numerically compare the observed IR and X-ray flare emission to our spherical source model by adapting the publicly available synchrotron-SSC code of Krawczynski et al. (2004). This code assumes a spherical emission region of radius $R$ moving at an angle $\theta$ from the observer's line of sight at speed $\beta=$ $v / c$, yielding the conventional Doppler parameter $\delta=1 /[\gamma(1-$ $\beta \cos \theta)]$. The electron spectrum is specified as a broken powerlaw distribution, and the density, magnetic field, and Doppler factor are also free. We have modified the code in small ways to suit our Galactic application, rather than the modeling of highenergy blazar spectra that led to its development. In Figure 7 we show three models that match the IR and X-ray spectra in the 2005 and 2006 flares. The parameters for the models are shown in Table 1. The model is underconstrained by the available data, so we hold 
TABLE 1

Synchrotron/SSC Models for the 2005 AND 2006 Flares

\begin{tabular}{|c|c|c|c|}
\hline Model & $\begin{array}{c}B \\
(G)\end{array}$ & $\begin{array}{c}R \\
\left(r_{\mathrm{S}}\right)\end{array}$ & $\begin{array}{c}n_{e} \\
\left(\mathrm{~cm}^{-3}\right)\end{array}$ \\
\hline$\ldots \ldots \ldots \ldots \ldots \ldots$ & 1.5 & 1.0 & $2.0 \times 10^{9}$ \\
\hline 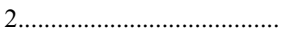 & 1.5 & 1.4 & $7.0 \times 10^{8}$ \\
\hline 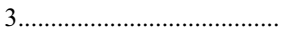 & 1.5 & 5.0 & $1.6 \times 10^{7}$ \\
\hline
\end{tabular}

the Doppler factor $(\delta=1.8)$ and the range of electron energies $\left(\gamma_{\min }=1, \gamma_{\max }=3 \times 10^{4}\right)$ fixed. Models 1 and 2 match the 2006 flare at its IR/X-ray peak (where the peak IR flux densities are assumed to match those of the 2005 flare) and near the start of the IR data, respectively. These differ by a 1.4 times adiabatic expansion, with the magnetic field strength held constant. Model 3 also reproduces the peak 2005 IR flux densities but evades the $\mathrm{X}$-ray upper limit because of its smaller optical depth to Compton scattering. The model parameters are plausible for Sgr A*, although polarization measurements likely prefer smaller densities (e.g., Marrone et al. 2007; Loeb \& Waxman 2007). This toy model is driven to higher densities by the need to reproduce the $\mathrm{X}$-ray emission entirely through self-Compton scattering; a more complete model of Sgr A* would include the quiescent submillimeter emission, and these additional seed photons and scattering electrons would therefore permit a smaller flaring density.

The delay between the flare times at optically thin and thick frequencies depends on the initial optical depth $\left(\tau_{\nu, 0}\right)$ at the frequency of interest and its rate of change. The synchrotron opacity of the model sphere scales as (Rybicki \& Lightman 1979)

$$
\tau_{\nu} \propto n_{e} R B^{(2+p) / 2} \nu^{-(4+p) / 2}
$$

Calculation of a "lifetime" for this opacity requires that we introduce some relationship between the quantities in equation (5) and time. The simplest procedure is to impose a power-law dependence of the radius on time, $R \propto t^{\beta}$, as in van der Laan (1966), with the magnetic field and density at fixed energy (or energy density) scaling as $B \propto R^{k_{\mathrm{B}}} \propto t^{\beta k_{\mathrm{B}}}$ and $n \propto R^{k_{n}} \propto t^{\beta k_{n}}$. Inserting these equations into equation (5) and its derivative, we find that the opacity decreases according to

$$
\begin{gathered}
\tau_{\nu}=\tau_{\nu, 0} t^{\beta \mu} \\
\mu=1+\frac{p+2}{2} k_{\mathrm{B}}+k_{n} .
\end{gathered}
$$

In the case of the van der Laan (1966) model, $k_{\mathrm{B}}=-2$ and $k_{n}=$ $-2-p$, so $\mu=-(3+2 p)$. Then the time $T_{\nu}$ required to reduce the source opacity to unity, the delay between the initial flare and the peak at frequency $\nu$, is

$$
T_{\nu}=\tau_{\nu, 0}^{-1 / \beta \mu}
$$

Combining equations (5) and (7), the delay depends on the initial parameters of the source according to

$$
\begin{aligned}
T_{\nu} & \propto\left[n_{e, 0} R_{0} B_{0}^{(p+2) / 2} \nu^{-(4+p) / 2}\right]^{-1 / \beta \mu} \\
& \propto\left[\frac{S_{\nu}^{\mathrm{IC}}}{S_{\nu}^{S}} B_{0}^{(p+2) / 2} \nu^{-(4+p) / 2}\right]^{-1 / \beta \mu}
\end{aligned}
$$

We can use the observed X-ray and IR flux densities in place of $S_{\nu}^{\mathrm{IC}}$ and $S_{\nu}^{S}$; normalization factors that depend on frequency in equations (3) and (4) will cancel in comparisons between flares because the observing wavelengths do not vary.

The two flares presented here can be used to examine the plausibility of this relationship. However, because we can only compare these flares through ratios of their properties, we cannot test the model without additional observations of flares having submillimeter and X-ray and/or IR counterparts. Normalized to the quiescent X-ray flux of Sgr A*, the 2006 flare represented a factor of 20 increase, while the 2005 flare produced $\leq 1.2$ times the quiescent flux (H07). The observed IR $\left(K^{\prime}\right)$ peak flux density was $12 \mathrm{mJy}$ in 2005 and $7 \mathrm{mJy}$ in 2006, but it is possible that the IR flux density was comparable to or even greater than $12 \mathrm{mJy}$ in 2006 before the observations began. Then $S_{\mathrm{X}, 2006} / S_{\mathrm{X}, 2005} \geq 20$, while $S_{\mathrm{IR}, 2006} / S_{\mathrm{IR}, 2005} \geq 0.6$. The ratio of the submillimeter delays is $T_{2006} / T_{2005}=4.8$. Assuming that the flares are created with similar magnetic field strengths, these ratios imply an upper limit on $\beta \mu$ of -2.2 . This decreases to -2.6 if we assume that the $\mathrm{X}$-ray emission is a factor of 2 below the upper limit. Reversing the argument, the weakest X-ray flares that can be reliably detected correspond to an excess of approximately twice the quiescent flux. Eckart et al. (2004) observed such a flare accompanied by a $4 \mathrm{mJy}$ IR flare ( $K$ band). Based on the parameters of the 2006 flare and this upper limit on $\beta \mu$, we expect that flares with IRsubmillimeter delays smaller than 50 minutes should not show measurable X-ray emission. Flares detected in the ongoing coordinated monitoring campaigns should be able to test this relationship in detail.

A relationship between the ratio of X-ray and IR fluxes and the submillimeter delay is expected even if the expansion of the flaring region does not follow the power-law form assumed above. An example is a plasma region expanding along a jet governed by the equations of Falcke \& Markoff (2000), which account for the acceleration due to the pressure gradient. Because the synchrotron opacity and the X-ray/IR flux ratio are proportional to the column density $\left(n_{e} R\right)$ of the plasma, the connection is imposed by assumption and the physics of the expansion merely determine the form of the correlation within the limits imposed by magnetic field variability.

\section{SUMMARY}

We have reported the first measurements of a flare of Sgr A* observed at submillimeter, IR, and X-ray wavelengths. Interestingly, the submillimeter flare is found more than an hour after the X-ray and IR flares. A large delay is also found between an IR and submillimeter flare in 2005, although the identification of the submillimeter flare with the IR event is less certain. We find the spectral and polarization changes in the flare to be consistent with expansion of a region of energetic plasma, although the adiabatic expansion model of van der Laan (1966) is not a good fit to this well-observed flare. Independent of the details of the expansion, this paradigm predicts that the delay between the submillimeter and short-wavelength flares should be related to the ratio of the synchrotron (IR) and SSC (X-ray) luminosities. Such a relationship should be testable in the ongoing multiwavelength Sgr A* monitoring campaigns. Campaigns including short-wavelength VLBI (1-7 mm) would also provide a test of the expansion model, as the long delays we observe imply expanded source sizes comparable to the measured intrinsic size (Shen et al. 2005; Bower et al. 2006). The IR and X-ray properties of the flares are well modeled by a homogeneous synchrotronSSC source, although incorporation of the submillimeter data likely requires a more detailed treatment. Incorporation of such 
expanding flares into the existing static models of Sgr A* will be essential for further progress in understanding the flares and the accretion region.

D. P. M. thanks Arieh Königl, John Raymond, and Brant Robertson for enlightening discussions. F. K. B. received support for this work from NASA through Chandra Award No. G05-6093X and G06-7041X, issued by the Chandra X-ray Center under contract NAS8-03060, and SAO Award No. 2834-MIT-SAO-4018. Some of these data were obtained at the W. M. Keck Observatory, which is operated as a scientific partnership among the California Institute of Technology, the University of California, and the National Aeronautics and Space Administration. The Observatory was made possible by the generous financial support of the W. M. Keck Foundation. The National Radio Astronomy
Observatory is a facility of the National Science Foundation operated under cooperative agreement by Associated Universities, Inc. The Submillimeter Array is a joint project between the Smithsonian Astrophysical Observatory and the Academia Sinica Institute of Astronomy and Astrophysics and is funded by the Smithsonian Institution and the Academia Sinica. The CSO is supported by the NSF under contract AST 05-40882. We thank David Chuss and Larry Kirby for assistance with the 2005 July observations at CSO. All of the ground-based data presented here were obtained from Mauna Kea observatories, a testament to the astronomical importance of this site. We are grateful to the Hawaiian people for permitting us to study the universe from this sacred summit.

Facilities: CXO (ACIS-I), Keck:II (NIRC2), CSO (SHARC-II), SMA (Polarimeter)
Aitken, D. K., Greaves, J., Chrysostomou, A., Jenness, T., Holland, W., Hough,

J. H., Pierce-Price, D., \& Richer, J. 2000, ApJ, 534, L173

Alexander, T. 1997, in Astronomical Time Series, ed. D. Maoz, A. Sternberg, \& E. M. Leibowitz (Dordrecht: Kluwer), 163

Baganoff, F. K., et al. 2001, Nature, 413, 45 2002, BAAS, 34, 1153 2003, ApJ, 591, 891

Bélanger, G., Goldwurm, A., Melia, F., Ferrando, P., Grosso, N., Porquet, D., Warwick, R., \& Yusef-Zadeh, F. 2005, ApJ, 635, 1095

Bélanger, G., Terrier, R., de Jager, O. C., Goldwurm, A., \& Melia, F. 2006, J. Phys. Conf. Ser., 54, 420

Bittner, J. M., Liu, S., Fryer, C. L., \& Petrosian, V. 2007, ApJ, 661, 863

Bloom, S. D., \& Marscher, A. P. 1996, ApJ, 461, 657

Bower, G. C., Falcke, H., Herrnstein, R. M., Zhao, J.-H., Goss, W. M., \& Backer, D. C. 2004, Science, 304, 704

Bower, G. C., Goss, W. M., Falcke, H., Backer, D. C., \& Lithwick, Y. 2006, ApJ, 648, L127

Bower, G. C., Wright, M. C. H., Falcke, H., \& Backer, D. C. 2003, ApJ, 588, 331

Broderick, A. E., \& Loeb, A. 2006, MNRAS, 367, 905

de Bruyn, A. G. 1976, A\&A, 52, 439

Dent, W. A. 1968, ApJ, 153, L29

Eckart, A., Schödel, R., Meyer, L., Trippe, S., Ott, T., \& Genzel, R. 2006a, A\&A, 455,1

Eckart, A., et al. 2004, A\&A, 427, 1 2006b, A\&A, 450, 535

- 2008, A\&A, 479, 625

Eisenhauer, F., Schödel, R., Genzel, R., Ott, T., Tecza, M., Abuter, R., Eckart, A., \& Alexander, T. 2003, ApJ, 597, L121

Eisenhauer, F., et al. 2005, ApJ, 628, 246

Falcke, H., \& Markoff, S. 2000, A\&A, 362, 113

Garmire, G. P., Bautz, M. W., Ford, P. G., Nousek, J. A., \& Ricker, G. R., Jr. 2003, Proc. SPIE, 4851, 28

Genzel, R., Schödel, R., Ott, T., Eckart, A., Alexander, T., Lacombe, F., Rouan, D., \& Aschenbach, B. 2003, Nature, 425, 934

Ghez, A. M., Becklin, E., Duchjne, G., Hornstein, S., Morris, M., Salim, S., \& Tanner, A. 2003, Astron. Nachr. Supp., 324, 527

Ghez, A. M., et al. 2004, ApJ, 601, L159 $2005, \mathrm{ApJ}, 635,1087$

Gillessen, S., et al. 2006, ApJ, 640, L163

Goldwurm, A., Brion, E., Goldoni, P., Ferrando, P., Daigne, F., Decourchelle, A., Warwick, R. S., \& Predehl, P. 2003, ApJ, 584, 751

Herrnstein, R. M., Zhao, J.-H., Bower, G. C., \& Goss, W. M. 2004, AJ, 127, 3399

Hornstein, S. D., Matthews, K., Ghez, A. M., Lu, J. R., Morris, M., Becklin, E. E., Rafelski, M., \& Baganoff, F. K. 2007, ApJ, 667, 900 (H07)

Königl, A. 1981, ApJ, 243, 700

Krabbe, A., Iserlohe, C., Larkin, J. E., Barczys, M., McElwain, M., Weiss, J., Wright, S. A., \& Quirrenbach, A. 2006, ApJ, 642, L145

\section{NCES}

Krawczynski, H., et al. 2004, ApJ, 601, 151

Krolik, J. H. 1999, Active Galactic Nuclei: From the Central Black Hole to the Galactic Environment (Princeton: Princeton Univ. Press)

Liu, S., Melia, F., \& Petrosian, V. 2006a, ApJ, 636, 798

Liu, S., Petrosian, V., Melia, F. \& Fryer, C. L. 2006b, ApJ, 648, 1020

Loeb, A., \& Waxman, E. 2007, J. Cosmol. Astropart. Phys., 3, 11

Markoff, S., Falcke, H., Yuan, F., \& Biermann, P. L. 2001, A\&A, 379, L13

Marrone, D. P. 2006, Ph.D. thesis, Harvard Univ.

Marrone, D. P., Moran, J. M., Zhao, J.-H., \& Rao, R. 2006a, ApJ, 640, 308

. 2006b, J. Phys. Conf. Ser., 54, 354 2007, ApJ, 654, L57

Mauerhan, J. C., Morris, M., Walter, F., \& Baganoff, F. K. 2005, ApJ, 623, L25

Melia, F., \& Falcke, H. 2001, ARA\&A, 39, 309

Melia, F., Liu, S., \& Coker, R. 2001, ApJ, 553, 146

Meyer, L., Eckart, A., Schödel, R., Duschl, W. J., Mužić, K., Dovčiak, M., \& Karas, V. 2006a, A\&A, 460, 15

Meyer, L., Schödel, R., Eckart, A., Karas, V., Dovčiak, M., \& Duschl, W. J. 2006b, A\&A, 458, L25

Pacholczyk, A. G. 1970, Radio Astrophysics (San Francisco: Freeman)

Pauliny-Toth, I. I. K., \& Kellermann, K. I. 1966, ApJ, 146, 634

Porquet, D., Predehl, P., Aschenbach, B., Grosso, N., Goldwurm, A., Goldoni, P., Warwick, R. S., \& Decourchelle, A. 2003, A\&A, 407, L17

Reid, M. J. 1993, ARA\&A, 31, 345

Rybicki, G. B., \& Lightman, A. P. 1979, Radiative Processes in Astrophysics (New York: Wiley-Interscience)

Shen, Z.-Q., Lo, K. Y., Liang, M.-C., Ho, P. T. P., \& Zhao, J.-H. 2005, Nature, 438,62

Shklovskii, I. S. 1960, Soviet Astron., 4, 243

Trippe, S., Paumard, T., Ott, T., Gillessen, S., Eisenhauer, F., Martins, F., \& Genzel, R. 2007, MNRAS, 375, 764

van Dam, M. A., et al. 2006, PASP, 118, 310

van der Laan, H. 1966, Nature, 211, 1131

Weisskopf, M. C., O’Dell, S. L., \& van Speybroeck, L. P. 1996, Proc. SPIE, 2805,2

Wizinowich, P., et al. 2000, PASP, 112, 315

Yuan, F., Quataert, E., \& Narayan, R. 2003, ApJ, 598, 301 2004, ApJ, 606, 894

Yusef-Zadeh, F., Roberts, D., Wardle, M., Heinke, C. O., \& Bower, G. C 2006a, ApJ, 650, 189

Yusef-Zadeh, F., Wardle, M., Cotton, W. D., Heinke, C. O., \& Roberts, D. A. 2007, ApJ, 668, L47

Yusef-Zadeh, F., Wardle, M., Heinke, C. O., Dowell, C. D., Roberts, D., Baganoff, F. K., \& Cotton, W. 2008, ApJ, 682, 361

Yusef-Zadeh, F., et al. 2006b, ApJ, 644, 198

Zhao, J.-H., Herrnstein, R. M., Bower, G. C., Goss, W. M., \& Liu, S. M. 2004, ApJ, 603, L85 